\title{
Influencia del tipo de centro educativo en las actitudes de los jóvenes hacia el dopaje
}

\section{Influence of the type of educational center on the attitudes of young people towards doping}

\author{
Gerardo José Ruiz-Rico Ruiz*, Félix Zurita Ortega², Manuel Castro Sánchez², Ramón Chacón Cuberos², \\ Javier Cachón Zagalaz ${ }^{3}$ y Pedro Ángel Valdivia Moral ${ }^{2}$ \\ 1 Universidad de Almería. \\ 2 Universidad de Granada. \\ 3 Universidad de Jaén.
}

\begin{abstract}
Resumen: El fenómeno del dopaje no solo está instaurado en el mundo del deporte profesional, sino que está causando cada vez más daño dentro del deporte amateur y recreacional. Ya que la edad de las personas que lo utilizan va disminuyendo con el tiempo, la presente investigación plantea como objetivo analizar y establecer relaciones entre las actitudes de jóvenes escolarizados hacia el dopaje, en función del tipo de centro (público o concertado/ privado) en donde estudian los jóvenes. En esta investigación, participaron 839 estudiantes de edades comprendidas entre los 15 y 18 ańos $(M=16.22$ años, DT=.77), que permitió el registro y evaluación de las variables tipo de centro escolar y actitudes hacia el dopaje, para la recogida de datos se utilizó la escala de actitudes hacia el dopaje (PEAS) de Petróczi (2002). Los resultados revelaron que la mayoría de los participantes tienen actitudes que rechazan el dopaje $(64,2 \% ; n=539)$; los alumnos que estaban escolarizados en centros públicos tienen actitudes más vulnerables hacia el dopaje $(\mathrm{p}=.014)$. Palabras clave: Dopaje. Actitudes. Permisividad. Centro Escolar.
\end{abstract}

Abstract: The phenomenon of the doping is not only instated in the world of the professional sport, also it is damaging increasingly inside the sport amateur and recreational. As the age of the persons who use it is diminishing along the time, the present investigation considers as aim analyzing and establishing relations between the young students' attitudes about the doping, depending on the type of school (public or concert / private) where young people study. In this investigation, 839 students between 15 and 18 years participated $\operatorname{in}(\mathrm{M}=16.22$ years, $\mathrm{DT}=.77)$, it allowed the record and evaluation of the variables type of school center and attitudes towards the doping, for his data collect we use the scale of attitudes towards the doping (PEAS) of Petróczi (2002). The results revealed that the majority of the participants have attitudes that reject doping $(64,2 \%$; $=539)$; the pupils who were schooled in public centers have more vulnerable attitudes towards the doping $(\mathrm{p}=.014)$.

Keywords: Doping. Attitudes. Permissiveness. School Center.

\section{Introducción}

El fenómeno del dopaje ha estado presente en nuestra sociedad desde hace siglos, está considerado perjudicial para la salud y además ha sido un lastre para el deporte y para la sociedad, ya que atenta contra la ética y la deportividad de éste (Atienza, 2013).Podemos definirlo como el uso de un método o de una sustancia utilizada como parte de una estrategia para lograr un fin que va en contra de las reglas, y a la que se puede recurrir con objetivos competitivos, estéticos y de rehabilitación (World Anti-Doping Agency, 2015).

En la actualidad nos encontramos con que, mucha población joven, además de deportistas profesionales, amateur y recreacionales, están recurriendo cada vez más al uso de sustancias dopantes. Por un lado, observamos que cada vez se dan más casos de deportistas profesionales que utilizan estos métodos como medio para poder llegar a conseguir sus metas deportivas. Esto puede ser debido a la gran exigencia

Dirección para correspondencia [Correspodence address]: Gerardo José Ruiz-Rico Ruiz. Universidad de Almería. E-mail: gerardo121988@ hotmail.com que existe en el deporte de alta competición, en el que cada milésima de segundo cuenta (Foucart, Verbanck \& Lebrun, 2015). Por otro lado, este comportamiento lo podemos observar de una forma más destacada en los usuarios de las salas de musculación o de los gimnasios, los cuales acuden en muchos casos a estas sustancias con el propósito de conseguir un mayor volumen de masa corporal o de músculo (Giraldi, Unim, Masala, Miccoli \& La Torre, 2015; Stubbe, Chorus, Frank, Hon \& Heijden, 2014; Rane, Rosen, Skarberg, Heine \& Ljungdahl, 2013).

Según algunos autores como Barkoukis, Tsorbatzoudis y Rodafinos (2013) las actitudes personales tienen un papel muy importante en este fenómeno ya que las intenciones de utilizar el dopaje están afectadas, por un lado, por causas proximales, como puede ser el control conductual, las reglas y actitudes, y por otro, por influencias distales, como son las orientaciones de meta. Por lo tanto, observaron que finalmente las actitudes son un factor principal en el propósito de doparse (Elbe \& Brand, 2016; Blank, 2015).

Otros autores como Morente-Sánchez y Zabala (2015) apreciaron en su investigación que gran parte de los miem- 
bros del personal técnico de los equipos de futbol españoles tenían actitudes y creencias muy tolerantes con el dopaje además de muy poco conocimiento sobre éste. Por lo tanto, estos autores evidenciaron la necesidad de implantar programas de intervención en contra del dopaje como instrumento fundamental de protección.

Como vemos existe una gran cantidad de investigaciones como la llevada a cabo por Gucciardi, Jalleh y Donovan (2011), en la que se destaca que el riesgo de consumir sustancias dopantes al que se ve sometido el deportista, en el que influyen sus actitudes, conocimientos y otras variables, puede llegar a ser mucho menor si se da una prevención incidiendo en las cuestiones legítimas y morales, que son las que a su vez influyen en las actitudes de éstos (Castro et al., 2015; Chacón et al., 2016; Elbe \& Brand, 2016; Espejo et al., 2017; Madigan, Stoeber \& Passfield, 2016; Whitaker, Backhouse \& Long, 2014). En esta misma línea Backhouse, Whitaker y Petroczi (2013), afirman que el riesgo de doparse de los deportistas que han realizado tareas cuyo objetivo era mejorar el cumplimiento de las normas, es mucho menor.

Igualmente, existen elementos que pueden ser determinantes en el nivel de permisividad que la persona tenga hacia el dopaje o hacia sustancias nocivas para su salud. De esta manera, en la investigación llevada a cabo por Dorr (2009) se hizo énfasis en el tipo de centro escolar en el que estén matriculados los jóvenes, como factor de riesgo a la hora de consumir ciertas sustancias nocivas para su salud. En esta investigación se llegó a la conclusión de que la probabilidad de consumir una sustancia como la marihuana era mayor en los centros públicos que en los concertados/privados.

Del mismo modo, autores como Morales, Ariza, Nebot, Pérez y Sánchez (2008) indagan sobre esta misma temática en su estudio, en el que participaron 2.043 alumnos de $3^{\circ}$ de la ESO pertenecientes a 47 centros escolares de Barcelona, y comprueban que el consumo de cannabis es mayor en jóvenes escolarizados en centros concertados/privados.

Por otra parte, Galán et al. (2012), realizaron un estudio que va en el mismo sentido que los anteriores, pero relacionando esta variable con el consumo de tabaco. En éste participaron 3622 alumnos de 79 escuelas de Madridy se concluyó igualmente que los alumnos escolarizados en las escuelas públicas eran más propensos a consumir tabaco (Barreto et al., 2010). Sin embargo hay investigaciones como la llevada a cabo por Campo-Arias, Ceballos y Herazo (2009) que contradicen a las anteriores, llegando a la conclusión de que el consumo de tabaco es mayor en jóvenes que están escolarizados en escuelas privadas.

En el presente estudio se aportan datos que confirman la relevancia que tienen estos factores en las actitudes de los jóvenes hacia el consumo de sustancias dopantes. A causa de esto, se plantean los siguientes objetivos para la presente investigación:
- Conocer las actitudes hacia el dopaje del alumnado del penúltimo curso de enseñanza no universitaria ( $1^{\circ} \mathrm{de}$ Bachillerato), y el tipo de centro en el que cursan sus estudios.

- Analizar las relaciones existentes entre las actitudes hacia el dopaje en estudiantes preuniversitarios, y el tipo de centro en el que cursan sus estudios.

\section{Método}

Diseño

El diseño de esta investigación consiste en un estudio empírico descriptivo de carácter cuantitativo puesto que su objeto principal es el de averiguar y analizar los diferentes datos que se obtengan sobre las variables y la relación entre ellas. Por otro lado, la parte cualitativa de la investigación se obtiene de los datos socio-demográficos que se han obtenido de la muestra.

\section{Participantes}

Para llevar a cabo este estudio se ha contado con una muestra de 839 de los cuales 390 eran chicos $(46,5 \%)$ y 449 eran chicas $(53,5 \%)$ con edades comprendidas entre 15 y 23 años $(M=16.22$ años, $D T=.77)$, estudiantes de $1^{\circ}$ de Bachillerato de 9 centros de la provincia de Jaén. La muestra se ha extraído de forma aleatoria de zonas urbanas (Jaén capital) y rurales (Torredelcampo y Torredonjimeno), 636 de la ciudad de Jaén, 121 de Torredelcampo y 82 de Torredonjimeno.Los centros educativos y estudiantes colaboraron voluntariamente en el desarrollo de la investigación. Fue necesario el consentimiento oficial de cada centro educativo para la puesta en marcha de este estudio.

\section{Variables e instrumentos}

En este estudio de investigación se tomó como referencia las siguientes variables e instrumentos:

- Tipo de Centro según público o concertado/privado

- Escala de actitudes hacia el dopaje, se recoge del cuestionario original "Performance Enhancement Attitude Scale (PEAS)”, creado originalmente por Petróczi (2002) y traducido y validado al español por Morente, Femia-Marzo y Zabala (2014). Es un instrumento unidimensional de autoinforme que contiene 17 ítems. Utiliza un formato de respuesta con escala tipo Likert, con 6 opciones de respuesta que van desde totalmente en desacuerdo (1), claramente en desacuerdo (2), algo en desacuerdo (3), algo de acuerdo (4), de acuerdo (5) a totalmente de acuerdo (6). Es significativo que no contiene una opción de respuesta neutral y todas las 
preguntas se realizan en la misma dirección. La puntuación oscila entre los rangos del 17 al 102. Cuando la puntuación de un individuo está por debajo del 59.5 indica que su actitud es menos favorable en general hacia el dopaje. Sin embargo, si es superior a 59.5, está indicando que la actitud de ese individuo es significativamente favorable al dopaje. Se determinó una fiabilidad de $\alpha=0,762$ en el presente trabajo.

\section{Procedimiento}

Para la recogida de datos, en primer lugar se elaboró una lista de los IES de la provincia de Jaén en los que se imparte Bachillerato, puesto que los alumnos a los que se les iba a aplicar el estudio forman parte de ese nivel de enseñanza. Posteriormente se eligieron de manera aleatoria los centros en los que íbamos a aplicar la toma de datos a través de los cuestionarios e indagamos sobre la ubicación y las características de cada uno de ellos. Conocida esta información, nos pusimos en contacto con los Directores de cada uno de estos centros. En las reuniones que mantuvimos con los Directores se incidió en la importancia del estudio para mejorar la salud de las personas, y la facilidad con la que se rellena el cuestionario, además de la corta duración de su cumplimentación. Los investigadores estuvieron presentes durante la recogida de los datos, informando sobre la mejor manera de rellenar los cuestionarios y asegurando el anonimato de las respuestas por parte de los investigadores.

\section{Análisis de los Datos}

El análisis de los datos se llevó a cabo con el programa estadístico SPSS, en su versión 21. La realización de los correspondientes análisis estadísticos se basó en el cálculo de los estadísticos descriptivos (medias y desviaciones típicas) con respecto al instrumento PEAS. Posteriormente se realizaron pruebas de diferencia de medias en aquellas varia- bles con dos alternativas de respuesta, y análisis de varianza ANOVA.

\section{Resultados}

En este estudio realizado sobre 839 jóvenes presenta una muestra heterogénea en cuanto al tipo de centro en el que cursaban sus estudios $(72,5 \%$; $\mathrm{n}=608$ pertenecían a un centro público, 27,5\%; n=231 a un centro concertado/privado). Respecto al nivel de permisividad hacia el dopaje, vemos que la gran mayoría de los participantes muestran un gran rechazo hacia éste ya que, como hemos podido observar, un $64,2 \%(n=539)$ tiene una mínima permisividad hacia el dopaje, un $32,9 \%(n=276)$ tiene poca permisividad, frente al $2,9 \%(\mathrm{n}=24)$ que la tienen alta, por lo que éste último sí que tiene una gran aceptación hacia el uso de sustancias dopantes.

Tabla 1. Descriptivos de la muestra.

\begin{tabular}{lll}
\hline Tipo de Centro & Público & $72,5 \%(\mathrm{n}=608)$ \\
& Concertado/Privado & $27,5 \%(\mathrm{n}=231)$ \\
\hline Nivel de Permisividad & Mínima Permisividad & $64,2 \%(\mathrm{n}=539)$ \\
al Dopaje & Poca Permisividad & $32,9 \%(\mathrm{n}=276)$ \\
& Alta Permisividad & $2,9 \%(\mathrm{n}=24)$ \\
\hline
\end{tabular}

Al relacionar el nivel de permisividad hacia el dopaje de los participantes de esta investigación, con el tipo de centro en el que éstos han cursado sus estudios, se comprueba que existen diferencias estadísticamente significativas $\left(p=.014^{* * *}\right)$, encontrando que los participantes que pertenecían a centros públicos obtienen mayores puntuaciones en las categorías "mínima permisividad" y "alta permisividad" invirtiéndose esta situación en la categoría "poca permisividad”(40,3\% frente al $30,1 \%)$, en las que las puntuaciones de los jóvenes que pertenecían a centros públicos son más bajas, observándose disparidad en la siguiente tabla (tabla 2).

Tabla 2. Nivel de permisividad hacia el dopaje según el tipo de centro donde se cursan los estudios.

\begin{tabular}{lllll}
\hline & \multicolumn{4}{c}{ Tipo de Centro } \\
\cline { 2 - 5 } Nivel de Permisividad al Dopaje & Público & Privado & Sig. \\
& Mínima Permisividad & $66,6 \%(\mathrm{n}=405)$ & $58 \%(\mathrm{n}=134)$ & .014 \\
& Poca Permisividad & $30,1 \%(\mathrm{n}=183)$ & $40,3 \%(\mathrm{n}=93)$ & \\
& Alta Permisividad & $3,3 \%(\mathrm{n}=20)$ & $1,7 \%(\mathrm{n}=4)$ & \\
\hline
\end{tabular}

\section{Discusión}

En esta investigación llevada a cabo a 839 estudiantes se ha manejado el mismo cuestionario que en los estudios realizados por Morente-Sánchez, Mateo-March y Zabala (2013),
Yager y O’Dea (2014) y Morente-Sánchez y Zabala (2015). Hemos podido observar al igual que en otros estudios anteriores al nuestro, (Whitaker, Backhouse y Long, 2014; RuizRico, Cachón, Valdivia y Zagalaz, 2014; Backhouse, Whitaker y Petroczi, 2013; Chan et al. 2015), que las actitudes 
tienen un papel muy importante a la hora de tomar la decisión de consumir sustancias dopantes.

Por otro lado, hemos podido apreciar que dentro de estas actitudes existen diferencias entre los alumnos en función de sí su centro escolar es público o concertado/privado. Los resultados nos indican que existe una diferencia muy significativa entre éstos, siendo los estudiantes que cursan sus estudios en centros públicos los que tienen un mayor porcentaje de éstos con una alta permisividad al dopaje. De la misma manera, estos resultados coinciden con los de otras investigaciones que preceden a la nuestra (Gooldberg et al., 2016; Azeredo, Levy, Peres, Menezes \& Araya, 2016; Martins-Oliveira, Jorge, Ferreira, Vale \& Zarzar2016) en las que se puede observar que existe un mayor número de conductas problemáticas o un mayor consumo de bebidas alcohólicas en jóvenes escolarizados en centros públicos.

Ante la imposibilidad de encontrar estudios que hayan analizado las actitudes hacia el dopaje de los adolescentes en función del tipo de centro escolar al que pertenecen, se analizan las actitudes hacia el consumo de otras sustancias prohibidas como las drogas. Uno de estos estudios, fue el llevado a cabo por Morales et al. (2008) en el que se obtuvieron resultados parecidos a los nuestros. En esta investigación participaron 2.043 alumnos de educación secundaria, de 47 escuelas de Barcelona. Estos autores llegaron a la conclusión de que existía una diferencia significativa $(\mathrm{p}=.01)$ entre el consumo de cannabis en los estudiantes de centros privados y centros públicos, siendo estos últimos los que tenían un mayor índice de consumo.

En un estudio de objetivos similares al nuestro, llevado a cabo por Lucchese, Burrone, Endersy Fernández (2013), se indagó sobre la importancia que puede tener el centro escolar en donde estén escolarizados los jóvenes a la hora de consumir sustancias prohibidas. En dicha investigación participaron 4593 alumnos de entre 13 y 17 ańos de edad, y en los resultados de ésta, se pudo apreciar que la probabilidad de consumir drogas como la marihuana o la cocaína era mucho mayor en jóvenes que pertenecían a centros públicos. Además también era mayor el consumo de tabaco en alumnos de este tipo de centros.

Sin embargo, en un estudio en el que participaron 54.675 escolares de Perú, llevado a cabo por Saravia, Gutiérrez y French (2014), se obtuvieron resultados que contradicen a las investigaciones mencionadas anteriormente. En éste se apreció que existía un 4\% más de probabilidad de iniciarse en el consumo de drogas en alumnos que estaban cursando sus estudios en colegios privados.
Después de haber observado los resultados obtenidos en esta investigación y haberlos comparado con los de las investigaciones expuestas anteriormente, pensamos que los jóvenes escolarizados en centros públicos tienen una mayor probabilidad de consumir sustancias dopantes en un futuro. Este fenómeno puede ser a causa de la falta de prevención que se debe de realizar en los centros educativos según la UNESCO (2005) en la Convención Internacional contra el dopaje en el deporte, en la que se aprobó, junto con la Agencia Mundial Antidopaje (AMA), la financiación de un proyecto para diseñar programas escolares de promoción de la ética deportiva y los valores de justicia en el deporte. Por otro lado, también puede ser debido a la repercusión que tiene el estrato socioeconómico sobre el consumo de este tipo de sustancias (Copeland \& Potwarka, 2015; Barkoukis, Tsorbarzoudis \& Rodafinos, 2013).

Como perspectivas de futuro de esta investigación podemos apreciar la necesidad de continuar investigando en esta línea con el objeto de identificar cuáles son los perfiles de jóvenes con actitudes más permisivas hacia el dopaje. Con esta información, y sabiendo que existen investigaciones como las llevadas a cabo por Elliot et al. (2004), Laure y Lecerf, (2002) y Goldberg et al. (2000), en las que se demuestra que a través de programas de intervención dentro de las clases de Educación Física se podría disminuir la intención de los jóvenes de consumir drogas, pensamos que se debería de trabajar en esta asignatura una línea que proponga educar sobre el tema y generar acciones tendientes a evitar o disminuir el uso de sustancias dopantes.

\section{Conclusiones}

Las conclusiones más relevantes a las que hemos llegado en esta investigación son, en primer lugar, que los alumnos preuniversitarios de la provincia de Jaén (España) tienen, en su mayoría, unas actitudes que rechazan el dopaje, aunque hemos podido observar que los alumnos que están escolarizados en centros públicos tienen una mayor tendencia al consumo de estas sustancias que los que pertenecen a un centro privado/concertado.

Los hallazgos a los que hemos llegado con este estudio nos ponen en evidencia la necesidad de incidir en la prevención al dopaje en alumnos escolarizados en centros públicos. Por otro lado, pensamos que la asignatura de Educación física podría ser un buen medio para conseguir esa prevención del consumo de sustancias dopantes.

\section{Bibliografía}

1. Atienza, E. (2013). Prevención, represión y control del dopaje frente al derecho a la intimidad del deportista en el marco jurídico español. Revista de Derecho Valparaiso, 40, 323-351.

2. Azeredo, C. M., Levy, R. B., Peres, M. F. T., Menezes, P. R., \& Araya,
R. (2016). Patterns of health-related behaviours among adolescents: a cross-sectional study based on the National Survey of School Health Brazil 2012. BMJ Open, 6(11), e011571.

3. Backhouse, S., Whitaker, L., \& Petroczi, A. (2013). Supplement use in 
the context of preferred competitive situations, doping attitude, beliefs, and norms. Scandinavian Journal Medicine \& Science Sports, 23, 244-252.

4. Barkoukis, V., Tsorbatzoudis, H., \&Rodafinos, A. (2013). Motivational and social cognitive predictors of doping intentions in elite sports: An integrated approach. Scandinavian Journal of Medicine \& Science in Sports, 23(5), 330-340.

5. Barreto, S. M., Giatti, L., Casado, L., Moura, L. D., Crespo, C., \& Malta, D. C. (2010). Smoking exposure among school children in Brazil. Ciencia \& saude coletiva, 15, 3027-3034.

6. Blank, C., Leichtfried, V., Shaiter, R., Fürhapter, C., \& Müller, D. (2015). Knowledge and attitudes among parents of Austrian junio athletes. Scandinavian Journal of Medicine \& Science in Sports, 25(1), 116-124.

7. Campo-Arias, A., Ceballos, G. A., \&Herazo, E. (2009). Consumo de Cigarrillo en estudiantes de una ciudad de Colombia: Factores asociados por género. Revista de Salud Pública, 11(4), 601-612.

8. Castro-Sánchez, M., Zurita-Ortega, F., Chacón-Cuberos, R., Martínez-Martínez, A., Espejo-Garcés, T., \& Álvaro-González, J. I. (2015). Harmful substances and motivational climate in relation to physical activity. Health and Addictions/Salud y drogas, 15(2), 115-126.

9. Chacón Cuberos, R., Castro Sánchez, M., Caracuel Cáliz, R., Padial Ruz, R., Collado Fernández, C., \& Zurita Ortega, F. (2016). Profiles of alcohol and tobacco use among adolescents from Andalusia in the first cycle of secondary education. Health and Addictions/Salud y Drogas, 16(2), 93-104.

10. Chan, D., Dimrnock, J., Donovan, R., Hardcastle, S., Lentillon-Kaestner, V., \& Hagger, M. (2015). Self-determined motivation in sport predicts anti-doping motivation and intention: A perspective from the trans-contextual model. Journal of Science and Medicine in Sport, 18(3), 315-322.

11. Copeland, R., \& Potwarka, L. (2016). Individual and contextual factors in ethical decision making: A case study of the most significant doping scandal in Canadian university sports history. Sport Management Review, 19(1), 61-68.

12. Dorr, A., Elena Gorostegui, M., Viani, S., \& Paz Dorr B, M. (2009). Teenage consumers of marijuana: implications for the family and the school. Salud Mental, 32(4), 269-278.

13. Elbe, A., \& Brand, R. (2016). The Effect of an Ethical Decision-Making Training on Young Athletes' Attitudes Toward Doping. Ethics \& Behavior, 26(1), 32-44.

14. Espejo Garcés, T., Martínez Martínez, A., Chacón Cuberos, R., Zurita Ortega, F., Castro Sánchez, M., \& Cachón Zagalaz, J. (2017). Alcohol consumption and physical activity in adolescents from rural environment. Health and Addictions/Salud y Drogas, 17(1), 97-105.

15. Elliot, D., Goldberg, L., Moe, E., DeFrancesco, C., Durham, M., \&Hix-Small, H. (2004). Preventing Substance Use and Disordered Eating Initial Outcomes of the ATHENA (Athletes Targeting Healthy Exercise and Nutrition Alternatives). Archives of Pediatrics \& Adolescent Medicine, 158(11), 1043-1049.

16. Foucart, J., Verbanck, P., \& Lebrun, P. (2015). Dopage, pratique sportive et addiction--quels liens? RevueMedicale de Bruxelles, 36(6), 485-493.

17. Galán, I., Díez-Gañán, L., Mata, N., Gandarillas, A., Cantero, J. L., \&Durbán, M. (2011). Individual and contextual factors associated to smoking on school premises. Nicotine \& Tobacco Research, ntr174.

18. Giraldi, G., Unim, B., Masala, D., Miccoli, S., \& La Torre, G. (2015). Knowledge, attitudes and behaviours on doping and supplements in young football players in Italy. Public Health, 129(7), 1007-1009.

19. Goldberg, L., MacKinnon, D., Elliot, D., Moe, E., Clarke, G., \& Cheong, J. (2000). The Adolescents traineng and learning to avoid steroids program preventing drug use and promoting health behaviors. Peditrics \& Adolescent Medicine, 154(4), 332-338.

20. Goldberg-Looney, L. D., Sánchez-SanSegundo, M., Ferrer-Cascales, R., Albaladejo-Blazquez, N., \&Perrin, P. B. (2016). Adolescent alcohol use in Spain: connections with friends, school, and other delinquent behaviors. Frontiers in psychology, 7 .
21. Gucciardi, D., Jalleh, G., \& Donovan, R. (2011). An examination of the Sport Drug Control Model with elite Australian athletes. Journal Science Medicine Sport, 14(6), 469-476.

22. Laure, P., \& Lecerf, T. (2002). Doping prevention among young athletes: comparison of a health education-based intervention versus information-based intervention. Science \& Sports, 17(4), 198-201.

23. Lucchese, M., Burrone, M. S., Enders, E. J., \& Fernández, A. R. (2013). Consumo de sustancias y escuela: un estudio en adolescentes escolarizados de Córdoba. Revista de salud pública, 17(1), 32-41.

24. Madigan, D., Stoeber, J., \&Passfield, L. (2016). Perfectionism and attitudes towards doping in junior athletes. Journal of Sports Sciences, 34(8), 700-706.

25. Martins-Oliveira, J. G., Jorge, K. O., Ferreira, R. C., Vale, M. P., \& Zarzar, P. M. (2016). Risk of alcohol dependence: prevalence, related problems and socioeconomic factors. Ciência \& Saúde Coletiva, 21(1), 17-26.

26. Morales, E., Ariza, C., Nebot, M., Pérez, A., \& Sánchez, F. (2008). Cannabis consumption among secondary school pupils of Barcelona [Spain]: initial use, reported effects and expectancies. Gaceta Sanitaria, 22(4), 321-329.

27. Morente-Sanchez, J., \& Zabala, M. (2015). Knowledge, attitudes and beliefs of technical staff towards doping in Spanish football. Journal of Sports Sciences, 33(12), 1267-1275.

28. Morente-Sanchez, J., Femia-Marzo, P., \& Zabala, M. (2014). CrossCultural Adaptation and Validation of the Spanish Version of the Performance Enhancement Attitude Scale (Petróczi, 2002). Journal Sports Science \& Medicine, 13(2), 430-438.

29. Morente-Sanchez, J., Mateo-March, M., \& Zabala, M. (2013). Attitudes towards Doping and Related Experience in Spanish National Cycling Teams According to Different Olympic Disciplines. PLOS one, 8(8), e70999.

30. Petroczi, A. (2002). . Exploring the doping dilemma in elite sport: Can athletes attitudes be responsible for doping? Colorado: University of Northem Colorado.

31. Rane, A., Rosen, T., Skarberg, K., Heine, L., \& Ljungdahl, S. (2013). Steroiderarettvaxandeproblempagymmen. Lakartidningen, 110(39-40), 1741-1746.

32. Ruiz-Rico, G., Cachón, J., Valdivia, P., \& Zagalaz, M. (2014). Dopaje Vs Fair play. Journal of Sport and Health Research, 1(6), 107-116.

33. Saravia, J. C., Gutiérrez, C., \& Frech, H. (2014). Factores asociados al inicio de consumo de drogas ilícitas en adolescentes de educación secundaria. Revista Peruana de Epidemiología, 18(1), 1-7.

34. Stubbe, J., Chorus, A., Frank, L., Hon, O., \& Heïjden, P. (2014). Prevalence of Use of Performance Enhancing Drugs by Fitness Centre Members. Drug Testing and Analysis(6), 434-438.

35. UNESCO. (19 de octubre de 2005). Conveción Internacional Contra el Dopaje en el Deporte. Recuperado el 23 de enero de 2018, de Convención Internacional Contra el Dopaje en el Deporte: http://portal.unesco. org/es/ev.php-URL_ID=31037\&URL_DO=DO_TOPIC\&URL SECTION=201.html

36. WADA. (21 de Febrero de 2014). World Anti-Doping Code. Obtenido de World Anti-Doping COde: http://www.wada-ama.org/Documents/ World_Anti-Doping_Program/WADP-The-Code/Code_Review/ Code\%20Review\%202015/Code\%20Final\%20Draft/WADA-Redline-2015-WADC-to-2009-WADC-EN.pdf

37. Whitaker, L., Backhouse, S., \& Long, J. (2014). Reporting doping in sport: National level athletes' perceptions of their role in doping prevention. Scandinavian Journal of Medicine \& Science in Sports, 24(6), 515-521.

38. Yager, Z., \& O’Dea, J. (2014). Relationships between body image, nutritional supplement use, and attitudes towards doping in sport among adolescent boys: implications for prevention programs. Journal of the International Society of Sports Nutrition, 11(1), 13. 